\title{
Getippte Dialoge in neuen Medien. Sprachkritische Aspekte und linguistische Analysen*
}

\author{
Christa Dürscheid/Sarah Brommer (Zürich)
}

\begin{abstract}
This article focuses on every day communication in New Media with special regards to private writing on Instant Messaging. After brief introductory thoughts about writings beyond the linguistic norm in New Media we compare the specific circumstances of "new" writing via internet and mobile phone with "traditional" offline writing that can be realized by the use of a computer, a type writer or by hand. How this new writing is judged by the public, whether it is considered to be "good" or "bad" and how experts position themselves in this discussion, is shown in section 3. Section 4 takes a look at which linguistic theories might apply to the analysis of typed dialogues in computer mediated communication. The main focus here is on the theory of Interactional Linguistics which formerly had been applied only to the analysis of oral communication. Finally, language critical and linguistic aspects of writing in the New Media are discussed in a brief synopsis.
\end{abstract}

Das Neue ist nicht schlecht, nur weil es eben neu ist. Freilich ist es auch nur darum nicht schon gut.

Hans-Martin Gauger (2004: 16)

\section{$1 \quad$ Alltagsschriftdeutsch}

"Millionenfach ergießt sich heute spontanes Alltagsschriftdeutsch [...] ins Internet. Dort lässt sich aufs ausführlichste besichtigen, wie es um die Schreibkompetenz der aus den Schulen ins Leben erlassenen Bevölkerung bestellt ist" (Zimmer 2005: 61). Mit diesen Worten kommentiert Dieter E. Zimmer in seinem Buch Sprache in Zeiten ihrer Unverbesserlichkeit das Schreiben im Internet. Als Basis für seine Beobachtungen dienen ihm 1'000 Sätze (aus politischen Diskussionsforen, aus Verkaufsangeboten auf der Online-Auktionsplattform $e$ Bay sowie aus verschiedenen Chaträumen), die nicht redigiert wurden und - zumindest nach erstem Augenschein - von Muttersprachlern stammen. Die Beispiele brauchen hier nicht im Einzelnen beschrieben zu werden; wer selbst gelegentlich Seiten im Internet aufsucht, weiß um die sprachlichen Auffälligkeiten in diesen Texten. Auch in der linguistischen Fachliteratur werden die Phänomene - meist unter dem Stichwort "konzeptionelle Mündlichkeit" ausführlich beschrieben, in der Regel aber nicht negativ-wertend dargestellt (vgl. stellvertretend für viele andere den Sammelband von Schlobinski 2006). Der Journalist Dieter E. Zimmer beschreibt diese Phänomene aber nicht nur, er urteilt auch über sie. So charakterisiert er Inflektivkonstruktionen vom Typ *verwirrtse ${ }^{*}$ und *einmalmitfreu* als "eklatante Systemverstöße, aber wenigstens von einem gewissen produktiven Sprachwitz" (2005: 63).

\footnotetext{
* Für wertvolle Hinweise zu diesem Beitrag danken wir Gerard Adarve und Andreas Gredig sowie zwei anonymen Gutachtern von Linguistik Online.
} 
Weiter spricht er von einer "Grammatik- und Orthographiewüste" (ibd.), führt Kurzschreibungen wie * $l \ddot{o} l^{*}$ und $* g^{*}$ an und fragt, wie sich in diesem "Kauderwelsch überhaupt einzelne Fehler bestimmen und zählen" (2005: 68) ließen. Eine Fehlerzählung nimmt er schließlich doch noch vor (z. B. "63 Prozent aller Sätze sind fehlerhaft; jeder von ihnen enthält im Durchschnitt 1,8 Fehler" (2005: 72f.)); sie bringt aber wenig Überraschendes und soll hier nicht Gegenstand weiterer Ausführungen sein. Die Frage, die in unserem Zusammenhang interessiert, ist eine andere.

Gesetzt den Fall, dass das "Alltagsschriftdeutsch" so normfern ist, wie Zimmer es beschreibt (und davon können wir in vielen Fällen ausgehen): Wie ist diese Tatsache zu bewerten? Welche Stellungnahmen finden sich dazu - und zwar nicht nur in der Öffentlichkeit, sondern auch in der Sprachwissenschaft? Denn wenn man davon ausgeht, dass es Aufgabe der Sprachwissenschaft ist, die Fragen und Bedürfnisse, die Menschen außerhalb des Fachs haben, ernst zu nehmen (vgl. Lanthaler et al. 2003: 5)1 ${ }^{1}$ dann kann man erwarten, dass sich die Sprachwissenschaft zu der Frage äußert, wie es in Anbetracht solcher Daten um die Schreibkompetenz bestellt ist und welche Folgerungen daraus $\mathrm{zu}$ ziehen sind. Diesen sprachkritischen Aspekten werden wir nachgehen und in Abschnitt 3 darstellen, welche Standpunkte sich dazu im wissenschaftlichen und öffentlichen Diskurs finden.

An dieser Stelle ist noch eine Bemerkung zum Schwerpunkt des vorliegenden Beitrags erforderlich: Zimmer konzentriert seine Beobachtungen auf Texte, die in Chatforen des Internets, in Blogs und eben auch in den vielen Annoncen auf $e B a y$ für alle einsehbar sind. In dieser Öffentlichkeit liegt aus seiner Sicht das eigentliche Problem: "Doch alle diese Botschaften waren ja keine Notizzettel, keine Mitteilungen an nächste Verwandte und engste Freunde. Sie waren für die anonyme Öffentlichkeit bestimmt" (2005: 77). Wir dagegen betrachten nur die nicht-öffentliche Kommunikation, also Äußerungen, die über SMS und in geschlossenen Chaträumen (z. B. im Instant Messaging) gemacht wurden. Hier lassen sich teilweise dieselben Phänomene finden, wie sie Zimmer in seiner Bestandsaufnahme konstatiert - und sie treten möglicherweise sogar noch stärker in den Vordergrund, weil es sich ausschließlich um getippte Dialoge handelt, um Äußerungen also, die in einem Kontext interaktiven Handelns stehen. ${ }^{2}$

Noch ein Wort zur Gliederung: In Abschnitt 2 werden die spezifischen Bedingungen des 'neuen' Schreibens via Internet und Handy mit dem 'alten', dem 'herkömmlichen' OfflineSchreiben, das sowohl am Computer, mit der Schreibmaschine als auch von Hand erfolgen kann, verglichen. Wie dieses neue Schreiben in der Öffentlichkeit beurteilt wird, ob es als "schlecht" oder als "gut" gilt (vgl. das einleitende Zitat von Hans-Martin Gauger) und wie sich die Wissenschaft in dieser Diskussion positioniert, ist Gegenstand von Abschnitt 3. Abschnitt 4 behandelt die Frage, welche linguistischen Theorien zur Analyse getippter Dialoge zur Verfügung stehen. In diesem Zusammenhang soll das Augenmerk insbesondere auf die Interaktionale Linguistik gerichtet werden, die sich bisher ausschließlich auf die Analyse mündlicher Interaktionen beschränkt. Abschließend werden die hier behandelten sprachkritischen und sprachwissenschaftlichen Aspekte zum Schreiben in den neuen Medien in einem knappen Resümee zusammengeführt.

\footnotetext{
${ }^{1}$ Wir beziehen uns hier auf Aussagen aus dem 'Bozner Manifest'. In diesem von fünf Sprachwissenschaftlern verfassten Text wird dafür argumentiert, dass die Sprachkritik nicht "selbst ernannten Experten" (Lanthaler et al. 2003: 3) überlassen werden dürfe, sondern als Teil einer anwendungsbezogenen Sprachwissenschaft betrieben werden müsse. Ihre Aufgabe sei es, Sprachgebrauchsphänomene kritisch zu reflektieren und Sprachbewertungsgrundlagen zu vermitteln.

2 Das trifft in Zimmers kleiner Studie nur für die Beispiele aus den Chatforen zu.
} 


\section{$2 \quad$ Neues Schreiben und neue Medien}

\subsection{Vorbemerkungen}

Der Titel 'Neues Schreiben und neue Medien' meint zweierlei: Zum einen soll damit angedeutet werden, dass sich mit den neuen Medien neue Kommunikationsformen entwickelt haben, die neue Formen des Schreibens hervorbringen. Zum anderen geht es um die Tatsache, dass Texte zunehmend mit Hilfe der neuen Medien erstellt werden und dieses Schreiben mit dem Arbeitsgerät Computer (und auch mit dem Handy) anderen Bedingungen unterliegt als das Schreiben von Hand. Hierunter fällt also alles, was mit der Nutzung und den Möglichkeiten von Rechtschreib- und Textverarbeitungsprogrammen (bspw. "cut and paste", Thesaurus, T9) und den Vor- und Nachteilen der Arbeit mit Tastatur und Bildschirm zu tun hat. Grundsätzlich liegt die Vermutung nahe, dass das Schreiben am Computer eine Veränderung des Schreibprozesses bewirkt hat, die mit den Veränderungen durch die Erfindung der Schreibmaschine vergleichbar ist (vgl. Spinnen 1992). ${ }^{3}$

Da wir das ganze Spektrum des Schreibens und seine verschiedenen Facetten darstellen wollen, seien zunächst einige grundlegende Aspekte zum Offline-Schreiben am Computer genannt. Im Anschluss daran werden wir auf die Besonderheiten des Online-Schreibens ${ }^{4}$ und des Schreibens am Handy eingehen.

\subsection{Schreiben am Computer (offline)}

Auch wenn das Tastaturschreiben selbst keine Errungenschaft des Computerzeitalters ist, unterscheidet sich das Tastaturschreiben am Computer in mehrfacher Hinsicht vom Schreiben mit der Schreibmaschine: Eine PC-Tastatur hat im Vergleich zu einer Schreibmaschinentastatur mehr Tasten und oftmals eine andere Zeichenbelegung. ${ }^{5}$ Zur Verwendung der Tastatur kommt die Bedienung einer Maus und die Tatsache hinzu, dass der Text beim Schreiben nicht auf Papier sichtbar ist, sondern auf dem Bildschirm. Darin besteht die zentrale Neuerung: Das Schreiben am Computer benötigt kein materiales, gegenständliches Trägermedium, wie dies beim Schreiben von Hand oder mit der Schreibmaschine der Fall ist. Durch den Computer als digitales Werkzeug ist es zudem möglich, über Tastenkombinationen Sonderzeichen darzustellen; auf der Schreibmaschine dagegen sind nur die Zeichen darstellbar, die auf der Tastatur auch vorgesehen sind. Der Schreiber hat also andere Möglichkeiten der Textgestaltung, als sie ihm auf einer herkömmlichen Tastatur zur Verfügung stehen.

Entscheidender aber noch sind die Möglichkeiten der Textverarbeitung, die die digitale Technik bereitstellt: Die Arbeit an einem Text kann an beliebiger Stelle aufgenommen und fortgesetzt werden. Hinzu kommt ein hohes Maß an Flexibilität durch die Möglichkeit des Löschens und Hin- und Herschiebens von Textblöcken. Weiter gestatten Textverarbeitungsprogramme wie Thesauri sowie Rechtschreib- und Grammatikhilfen das Experimentieren mit neuen Wörtern und Wortkombinationen und erlauben die schnelle Korrektur des verfassten Textes, ohne dass auf medienexterne Hilfsmittel zurückgegriffen werden muss. "Beim Computerschreiben hat man eine Art Bleistift mit eingebautem Radiergummi in der Hand,

\footnotetext{
${ }^{3}$ Das folgende Zitat verdeutlicht dies: "Mit der Einführung der elektronischen Datenübermittlung fern von Kugelschreiber, Filzstift, Füller und Schreibpapier haben sich die Schreibstile verändert" (Süddeutsche Zeitung 27. Oktober 1997).

${ }^{4}$ Damit ist nicht gesagt, dass die Schreiber beim Verfassen des Textes notwendigerweise online sind. So kann eine E-Mail durchaus auch offline geschrieben werden. Wichtig aber ist, dass die Datenübermittlung im selben Medium erfolgt wie das Verfassen des Textes.

${ }^{5}$ Eine feststehende Anordnung gibt es bei PC-Tastaturen nicht; jede Systemarchitektur hat hier gewisse Eigenheiten. Unterschiede ergeben sich insbesondere bei Notebooks durch das Platzproblem.
} 
Schere und Leim stehen bereit", hält Burkhard Spinnen (1992: 45) fest und fährt fort: "Der psychologische Unterschied zum Maschinenschreiben ist enorm; mußte dort jeder Anschlag sitzen, ist nun prinzipiell nichts ein Fehler." Das jeweilige Resultat ist zunächst nur virtuell vorhanden, als eine Summe von Bildpunkten auf dem Bildschirm. ${ }^{6}$ Das, was Hayes/Flower (1980: 11) als die drei Phasen der Textproduktion (planning, translating 7 , reviewing) bezeichnen, lässt sich so ohne großen Aufwand realisieren.

Daraus wiederum resultiert, dass Bottom-up-Strategien beim Schreiben zunehmen. Sprachliche Planungsprozesse finden demnach nicht vor der eigentlichen Textproduktion statt, sondern Textinhalt und -aufbau werden im Laufe des Schreibprozesses erst entwickelt. Ein systematischer Nachweis über diesen Effekt liegt bisher jedoch nicht vor, ebenso fehlen empirische Erkenntnisse über weitere Phänomene, die mit dem Schreiben am Computer verbunden sind. Dazu rechnen Eva-Maria Jakobs und Ines Merker-Melcher (2003: 832f.) den "höhere[n] Produktionsdruck, der zur Verkürzung von Planungsschritten führt", die "Beschleunigung des Schreibens bei geringerer Internalisierung des Geschriebenen" und die "Tendenz zu längeren Texten im Vergleich zur Bedingung 'Schreiben mit Papier und Bleistift'".

\subsection{Schreiben am Computer (online)}

Der spezifische Sprachgebrauch im Internet - sollte es einen solchen tatsächlich geben - liegt nicht an dem Schreiben via Tastatur, sondern an den Möglichkeiten der direkten Datenübermittlung. So kann ein Text in der Kommunikationsform E-Mail grundsätzlich auch ohne spezifischen Sprachgebrauch auskommen und sich in der Textform an einem herkömmlichen Brief orientieren. Neu ist aber bspw., dass in einer E-Mail im Unterschied zur 'Snail Mail' Textpassagen aus einer Bezugs-Mail übernommen werden können und dadurch eine Dialogstruktur entsteht. ${ }^{8}$ Dass sowohl die Produktion und die Distribution als auch die Rezeption des Textes in ein und demselben Medium stattfinden, bringt die schriftliche Interaktion in die Nähe des mündlichen Gesprächs; Angelika Storrer (2001) spricht in diesem Zusammenhang denn auch von "getippten Gesprächen". Wir dagegen ziehen die Bezeichnung "getippte Dialoge" vor, da bei diesem Schreiben in den neuen Medien trotz der Nähe zur medialen Mündlichkeit das wesentliche Merkmal von Gesprächen fehlt: Rezeption und Produktion der Äußerung verlaufen nicht simultan.

Die verschiedenen Formen des Online-Schreibens unterscheiden sich grundlegend vom konventionellen Offline-Schreiben per Hand oder Tastatur. So ermöglicht die schnelle Datenvermittlung neben der asynchronen Kommunikation (wie in der E-Mail) auch eine quasi-synchrone Kommunikation (wie im Chat). ${ }^{9}$ Während in der E-Mail-Kommunikation das zeitnahe Antworten zwar möglich, aber nicht konstitutiv ist, sind bei Chat- oder InstantMessaging-Dialogen Quasi-Synchronizität und Dialogizität die Grundlage der Kommunikation. Dies führt zu einem vermehrten Auftreten von Ausdrucksmitteln, die eher

\footnotetext{
${ }^{6}$ Daraus resultiert auch ein Nachteil, denn bekanntlich werden Fehler auf dem Bildschirm schneller überlesen als auf dem Papier; außerdem verlangt das Lesen am Bildschirm eine höhere Konzentration. Untersuchungen von Ziefle (1998) zufolge fallen Leseleistungen am Bildschirm unabhängig von äußeren Einflüssen wie Licht und Bildschirmauflösung immer bis zu zehn Prozent schlechter aus (vgl. Jakobs/Merker-Melcher 2003: 833).

7 "Translating" ist in diesem Zusammenhang als Formulierungsprozess zu verstehen, d. h. als das Übersetzen der entwickelten Schreibpläne in schriftliche Äußerungen.

${ }^{8}$ So kann man durch kurze Einschübe, die das Zitat unterbrechen, direkt auf einzelne Sequenzen antworten. Begrenzt besteht diese Möglichkeit auch bei der Faxkommunikation, indem die Antwort direkt auf das ausgedruckte Fax geschrieben wird.

${ }^{9}$ Daneben gibt es eine Chattechnologie, bei der der Leser den Schreibvorgang seines Kommunikationspartners unmittelbar verfolgen kann. Diese wechselseitige synchrone Kommunikationsform (vgl. Dürscheid 2004: 149-153) konnte sich aber nicht durchsetzen. Entsprechende Angebote für die Öffentlichkeit sind praktisch nicht mehr vorhanden.
} 
der medialen Mündlichkeit zuzuordnen sind (bspw. Gesprächspartikeln, Assimilations- und Reduktionsformen, Satzabbrüche, Interjektionen, umgangssprachliche Ausdrucksweisen und Dialektismen). Je "synchroner" die Kommunikation, desto stärker nähert sich der Sprachgebrauch der konzeptionellen Mündlichkeit an und desto eher entspricht der Sprachgebrauch dem, was gemeinhin als typisch für das Schreiben in den neuen Medien bezeichnet wird. Beobachten lässt sich dies beispielsweise an folgendem Instant-Messaging-Dialog zweier Studenten. ${ }^{10}$ An den Stellen, an denen der Beitrag von uns in a) und b) untergliedert wurde, haben die Verfasser den Text in zwei Etappen abgeschickt.
A: ok gestern habe ich mich ja ein bisschen aufgeregt bei dsds
B: löl warum?
A: weil 2 rausgefflogen sind die wirklich gut waren oder zumindest einiges besser als 2 die weiterkamen
B: a) ich fand $[x y]$ so ein mongo
b) er hats voll verdient
A: warum?
B: er ist einfach ein loser und ein mongo
A: a) ich fand den cool
b) der hat ne geile kopfstimme

Auffallend an dieser Sequenz ist neben den Merkmalen konzeptioneller Mündlichkeit, dass keine Satzzeichen gesetzt werden - abgesehen von den Fällen, in denen eine Frage gekennzeichnet wird. Diese Abweichung von der standardsprachlichen Norm ist keine Ausnahme: Florence Kessler (2008) hat in ihrer Untersuchung von 300 IM-Dialogen unter Studenten nachgewiesen, dass die typischen Phänomene der Medienkommunikation auch unter Studierenden auftreten, also bei Personen, die einen höheren Schulabschluss erreicht haben und denen in der Regel eine gewisse Schreibkompetenz zugesprochen wird. Normabweichendes Schreiben in der schriftlichen Alltagskommunikation geht demnach nicht zwangsläufig, wie so oft befürchtet (vgl. Abschnitt 3), mit mangelnder Schreibkompetenz in normgebunden Kontexten einher.

\subsection{Schreiben am Handy}

Neben dem Offline- und dem Online-Schreiben kommt ein dritter Aspekt in der Medienkommunikation hinzu, auf den gesondert eingegangen werden muss: das Schreiben am Handy. Die Zahlentastatur und das nur wenige Zentimeter große Handy-Display, aber auch die Beschränkungen in der Zeichenzahl schaffen hier spezifische Schreibbedingungen, die weder mit dem Computerschreiben noch mit dem handschriftlichen Schreiben vergleichbar sind. So kommen Buchstabendreher am ehesten in Texten vor, die am Computer geschrieben wurden; am Handy dagegen lassen sich Fehler konstatieren, die auf die Eingabe über die Worterkennungssoftware wie bspw. T9 oder das mehrmalige Drücken einer Taste zur Darstellung eines Buchstabens zurückzuführen sind:

(1) Hi du! Lass vor um neun in der Stadt treffen, ok? GuK

(2) Bir nachher! Lg!

Im ersten Fall wurde es versäumt, den ersten von T9 angebotenen Wortvorschlag vor zugunsten des richtigen uns (zweiter T9-Vorschlag) auszutauschen. Im zweiten Beispiel wurde bei "bir" die Taste 7 versehentlich nur dreimal gedrückt, mit der Folge, dass anstatt des richtigen "s" (= "bis") das "r" erschien. Neben diesen eingabebedingten Schreibweisen kann die beim SMS-Schreiben oft übliche Begrenzung auf 160 Zeichen zu verkürzten Schreib-

\footnotetext{
${ }^{10}$ Freundlicherweise hat uns Florence Kessler Auszüge aus ihrem Korpus zur Verfügung gestellt.
} 
weisen und elliptischen Satzkonstruktionen führen. Dies illustrieren die folgenden Beispiele, wo Kurzschreibungen wie "wg", "d" und "hdmg" stehen und elliptische Konstruktionen wie "weiss ned":

(3) Hallo [xy]-Schatz, beste Grüße aus SaasFee!I ruf Dich wg d Osterplanung am Mo an.Gruß an $[x y]$ u Kixx vom Fx

(4) Ey nad, du hesh KA.. :"o( mir gats so shaiize *cry* weiss ned was mache... hdmg kiizz Aa

In der kritischen Auseinandersetzung um das Schreiben in den neuen Kommunikationsformen wird deshalb besonders oft das SMS-Schreiben angeführt. Dies ist in der Tat das beste Beispiel, wenn man dafür argumentieren möchte, dass das Medienschreiben zu einem elliptischen Sprachgebrauch und zur Verwendung von Akronymen, Kurzwörtern und auch Rebusschreibungen (vgl. gute N8) führt.

\subsection{Schlussbemerkung}

Abschließend ist festzuhalten, dass man von der Kommunikationsform (Chat, E-Mail, SMS etc.) nicht ohne Weiteres auf das Medium (Computer, Handy) schließen kann: SMS gelten zwar gemeinhin als am Handy verfasste Texte, können aber auch via Internet verschickt und mit der Computertastatur geschrieben werden (dann ohne Texterkennungsprogramm und oft ohne Begrenzung des Zeichenumfangs). Umgekehrt gilt für E-Mails, IM-Texte und Blogbeiträge, dass sie - anders als bislang meist üblich - auch am Handy geschrieben werden können, sofern dieses über einen Internetbrowser verfügt. ${ }^{11}$ Das Spektrum der Textproduktion und -rezeption in Kombination mit den verschiedenen Medien ist also groß, und längst gilt nicht mehr die einfache Zuordnung von SMS zum Handy bzw. von der Chat- und E-MailKommunikation zum Computer. Ebenso ist die Trennung in öffentliche und private Kommunikation in Anbetracht der neuen Datenübermittlung zu überdenken: Denn mit der Infrastruktur des Netzes und der digitalen Kommunikation wird oftmals 'öffentlich', was eigentlich für private Zwecke bestimmt war (bspw. in der E-Mail-Kommunikation durch versehentliches Weiterleiten). Und auch der umgekehrte Fall kommt vor: "Auf der anderen Seite mag vieles zwar öffentlich sein, aber kaum Aufmerksamkeit erregen" (Bittner 2003: 292). Man denke hier nur an die vielen privaten Homepages.

\section{$3 \quad$ Sprachkritische Aspekte}

\subsection{Schreiben im Fokus der Sprachkritik}

In Zeiten von Schulleistungsstudien, Kompetenzmessungen und populären sprachkritischen Publikationen im Stil Bastian Sicks ${ }^{12}$ gelangt das Schreiben und hier insbesondere das Schreiben in den neuen Medien immer wieder in den Fokus kritischer Betrachtungen. Mehr noch als in der Wissenschaft wird denn auch in der Öffentlichkeit die Frage nach dem möglichen Einfluss der neuen Medien auf das Schreiben gestellt. Dies zeigt auch ein Blick in Zeitschriften und Zeitungen der vergangenen Jahre. ${ }^{13}$ So wird in der Frankfurter Allgemeinen Zeitung vom 31.07.2001 eine Entwicklung beklagt, die "die elektronische Textverarbeitung im Alltagsgebrauch ohnehin vorantreib[e]: Sprache wird zu einem Objekt der Beliebigkeit,

\footnotetext{
${ }^{11}$ So ermöglicht das mobile Gerät $O G O$ (u. a. von den Firmen lundl und Swisscom angeboten) neben der klassischen Handynutzung über einen Internet-Browser auch die Chat-, IM- und E-Mail-Kommunikation allerdings mit einer klassischen Alphabettastatur.

12 Vgl. dazu ausführlich Schneider (2005).

13 Die hier exemplarisch ausgewählten Zitate sind, abgesehen von drei neueren Artikeln, einem Korpus zum öffentlichen Diskurs über die Schreibkompetenz entnommen, das insgesamt 216 Texte aus den Jahren 1994 bis 2005 umfasst und auf der Auswertung von zehn deutschsprachigen Zeitungen und Zeitschriften basiert (vgl. Brommer 2006).
} 
das Schreiben schlampiger, das Lesen oberflächlicher". Und im Spiegel vom 5. Mai 2006 heißt es: "Im Internet tippt Otto-Normalsurfer so krumm, wie ihm die Finger gewachsen sind. Heraus kommen schräge Kurzformen, krude Schreibweisen und - freundlich gesagt lautmalerische Umschreibungen des Sinns."

Dass das Schreiben in den neuen Medien in der Öffentlichkeit so kritisch beäugt wird, hängt sicher auch damit zusammen, dass es inzwischen den Großteil der schriftlichen Alltagskommunikation darstellt. Zwar gibt es immer noch Bereiche, in denen handschriftlich kommuniziert wird (z. B. Postkarten, kurze Notizen für Partner oder Arbeitskollegen, Schülerzettel), doch sind diese marginal gegenüber der Kommunikation mittels Computer und Handy. Dabei ist es nicht nur so, dass die Kommunikation über Computer und Handy andere Formen schriftlicher Kommunikation ersetzt; hinzu kommt auch, dass sie in Situationen eingesetzt wird, wo früher ein persönliches Gespräch oder ein Telefonat geführt worden wäre. So berichten Mitarbeiter in großen Firmen, dass das Telefon praktisch nicht mehr läute, da der gesamte Nachrichtenverkehr über E-Mail oder in internen IM-Netzwerken abgewickelt werde. ${ }^{14}$

\subsection{Der öffentliche Diskurs}

Die Thematisierung der Schreibleistungen von Jugendlichen erfolgt im öffentlichen Diskurs, genauer in Zeitungen und Zeitschriften, in unterschiedlicher Intensität. ${ }^{15}$ Dass hier meist ein Zusammenhang zwischen den neuen Medien und dem Schreiben angenommen wird, zeigt sich, wenn von "spezifische[m] Sprachgebrauch" (FAZ 13.08.1997) und "veränderte[n] Schreibstile[n]" (SZ 27.10.1997) die Rede ist. Mit den neuen Kommunikationsformen habe sich nicht nur der "Prozess des Schreibens [...] grundlegend verändert" (Stern 20.01.2004), der "Sprachgebrauch im Web veränder[e] die Sprache selbst" (Spiegel 05.05.2006). Im Mittelpunkt des Interesses steht demnach der Einfluss der neuen Medien auf das Schreibverhalten. Dabei lässt sich diachron eine Erweiterung des Blickwinkels feststellen:

Ende der 1990er Jahre bezogen sich die Aussagen nur auf den Sprachgebrauch in den neuen Medien, inzwischen aber wird der Einfluss umfassender gesehen und die Veränderung nicht mehr an das Medium gebunden: "SMS, Chat und Internet hinterlassen ihre Spuren in der Sprache" (SoZ 18.11.2007). Anders gesagt: Während zunächst von einer spezifischen SMSoder Netzsprache ausgegangen wurde, herrscht inzwischen im öffentlichen Sprachbewusstsein die Meinung vor, dass der spezifische Sprachgebrauch nicht an das Medium gebunden sei. Dass der mediale Verwendungskontext entscheidend sein könnte, wird im öffentlichen Diskurs also weniger beachtet. Vielmehr ist den Zeitungs- und Zeitschriftenartikeln zu entnehmen, dass angenommen wird, die Eigenheiten der vermeintlichen Netzsprache seien inzwischen auch außerhalb des Netzes zu finden.

Übereinstimmend wird festgestellt, dass das Schreiben durch die neuen Medien quantitativ zugenommen habe: "Man schreibt wieder Briefe, wenn auch elektronische, kurz und auf englisch 'E-mail' genannt" (FAZ 13.08.1997), heißt es schon 1997, und noch 2005 bestätigt Bernd Eichinger in einem Artikel diese Entwicklung: Es "schreiben durch den Computer wesentlich mehr Menschen. Wer nie einen Brief schrieb, schreibt heute eine Mail oder eine SMS" (Stern vom 18.03.2005). Mit der quantitativen Zunahme des Schreibens wird auch das gängige Vorurteil, dass das Schreiben von E-Mail und SMS, in Chats und Foren qualitativ schlechter sei, relativiert (ZEIT 21.10.2004): "Wenn hie und da der Eindruck entsteht, man lese mehr Fehler als früher, hat das auch damit zu tun, dass heute mehr Leute schreiben als

\footnotetext{
${ }^{14}$ Vgl. hierzu Freyermuth (2002: 7), der diesen Umstand folgendermaßen kommentiert: "Anderen fernmündlich die eigene Zeit aufzuzwingen und ihnen so die ihre zu stehlen, gilt [...] in immer mehr Branchen und immer weiteren sozialen Kreisen als zunehmend unfein."

15 In den folgenden Ausführungen beziehen wir uns auf Brommer (2006), vgl. auch Brommer (2007).
} 
früher." Dass das Schreiben zugenommen hat, wird der erhöhten Sprachkreativität zugeschrieben, die aus der in den neuen Kommunikationsformen geringeren Normierung folge (ZEIT 20.08.1998): "Experimentierlust ohne Debatte über richtig oder falsch, das Netz eröffnet und seine Nutzer verteidigen diesen Freiraum." Der Betonung von Freiheit, Kreativität und Toleranz auf der einen Seite steht die Kritik an der vermeintlich zunehmenden Regellosigkeit und Verrohung des Sprachgebrauchs gegenüber. Insgesamt gesehen sind im öffentlichen Diskurs die medienpessimistischen Töne dominant. Die angenommene Verschlechterung der Schreibkompetenz wird häufig auf die Computer- und Internetnutzung zurückgeführt. Diese Aussage wird bevorzugt damit begründet, dass der Sprachgebrauch im Internet in orthographischer, grammatischer, lexikalischer und stilistischer Hinsicht defizitär sei und sich dies auf das herkömmliche Schreiben auswirke.

Grundsätzlich ist zu beobachten, dass die Auseinandersetzung mit dem Thema v. a. dann mit einer pessimistischen Einschätzung verbunden ist, wenn ein anderer Aspekt (bspw. der Lehrstellenmarkt) im Vordergrund steht. Steht hingegen das Schreiben selbst im Fokus der Betrachtung, fällt das Urteil weniger pessimistisch aus. Alles in allem ergibt sich somit kein einseitig negatives Bild, auch positive und optimistische Aussagen zum Schreiben in den neuen Kommunikationsformen sind im öffentlichen Diskurs vorhanden. Allerdings sind diese quantitativ stark unterrepräsentiert, so dass sich der negative Gesamteindruck durchsetzt unabhängig vom Zeitpunkt der Betrachtung.

\subsection{Der wissenschaftliche Diskurs}

Im Kontrast zur eher medienpessimistischen Einschätzung im öffentlichen Diskurs dominiert im wissenschaftlichen Diskurs die optimistische Ansicht. Dies mag einerseits damit zusammenhängen, dass sich der wissenschaftliche Diskurs als relativierende Gegenposition zum öffentlichen Diskurs versteht. Ein anderer Grund liegt vermutlich in dem problematischen Verhältnis der Sprachwissenschaft zur Sprachkritik, das weiter oben schon kurz angesprochen wurde. Hans-Martin Gauger (1999) ist in einem gleichnamigen Aufsatz dieser "Hilflosigkeit der Sprachwissenschaft" nachgegangen (vgl. auch Neuland 1996).

Schauen wir uns den wissenschaftlichen Diskurs nun aber genauer an: In vielen linguistischen Arbeiten stehen die Vorteile und der Nutzen, der aus den neuen Medien zu ziehen ist, im Vordergrund. Die neuen Medien würden mitnichten einen Sprachverfall verursachen, vielmehr bedeuten sie nach Wolfgang Raible (2001:22) "eine neue kommunikative Herausforderung, der wir sprachlich gerecht werden müssen. Auch sie werden zum Ausbau des Sprachsystems beitragen". Die These vom defizitären Schreiben in den neuen Kommunikationsformen wird in der Regel relativiert und die Normabweichungen als ein bewusstes Hinwegsetzen über die Normen interpretiert. Mit den Medien seien veränderte Kommunikationssituationen entstanden, an die eine Anpassung notwendig sei. Aus diesem Grund seien die Texte in den neuen Kommunikationsformen

keineswegs unverständlich, sondern Ausdruck funktionaler Schreibprozesse. Dies bedeutet nicht, dass der Schreiber nicht anders schreiben kann, sondern vielmehr, dass er so schreibt, wie es vor dem Hintergrund der technischen Voraussetzungen kommunikativ erforderlich ist. (Schlobinski 2003, o. S.)

Ein Text wird damit nicht aufgrund seiner Konformität mit den schulischen Normen, sondern an seiner situativen Angemessenheit beurteilt. Hierfür ist nicht allein die Kommunikationsform ausschlaggebend, sondern die Tatsache, an wen das Schreiben gerichtet ist, worin der Anlass des Schreibens besteht und wie sich die Kommunikationssituation als Ganzes gestaltet. Dass sich der wissenschaftliche Diskurs in seiner Argumentation dabei konsequent 
von der öffentlichen "Laien-Linguistik" abgrenzt, hat Tradition. ${ }^{16}$ Vermeintlicher Unwissenschaftlichkeit und Meinungsmache werden - vermeintliche - Objektivität und Neutralität entgegengesetzt. Wo die Sprachkritik wertet, will die Linguistik nur beschreiben. Doch "die Linguisten geben vielfach vor, sie würden nicht bewerten, tatsächlich bewerten sie aber doch", hält Gauger (1999: 99, Hervorh. im Orig.) fest, "die Linguisten finden nämlich immer alles prima, was sich in der Sprache ergeben hat." Gauger bezieht sich in seiner Kritik auf den Aufsatz "Sprachkritik und Sprachwissenschaft" von Peter von Polenz (1963), angesprochen ist jedoch jeder Linguist,

weil er in der Tat das faktisch Vorgefundene stets positiv bewertet, einfach, weil es da ist. Die Sprachwissenschaftler haben einen eigentümlichen Zwang, alles, was ist, zu rechtfertigen und zu zeigen, daß es gar nicht anders sein kann, als es ist. (Gauger 1999: 100)

Die fast ausnahmslos positive Beurteilung des Schreibens in den neuen Kommunikationsformen von Seiten der Wissenschaft im Sinne einer Bereicherung der bisherigen Ausdrucksmöglichkeiten darf also auch nicht unkritisch gesehen werden. Analog zu den Beobachtungen, die Jürgen Spitzmüller (2006) in Bezug auf die Darstellung von Jugendsprache im öffentlichen Diskurs angestellt hat, kann man von einer "Meliorisierung" der Mediensprache im wissenschaftlichen Diskurs sprechen. So gibt es nur wenige linguistische Arbeiten, die eine kritische Haltung gegenüber dem heutigen Sprachgebrauch der Schüler einnehmen. Dazu zählt ein Beitrag von Wilhelm Grießhaber, der sich aber nicht auf das Schreiben in den neuen Medien bezieht, sondern auf den Einfluss des ethnolektalen Deutsch. Grießhaber prognostiziert, dass sich das Deutsche zu einem "morphologisch reduzierten Deutsch" (Grießhaber 2007: 343) entwickle und Schüler, die über "die alten differenzierten Ausdrucksmittel" nicht mehr verfügten, "verbal eingeschränkter handlungsfähig" seien. Inwieweit diese Prognose zutrifft und ob Sprecher des "morphologisch reduzierten Deutsch" tatsächlich nicht in der Lage sind, komplexe Sachverhalte darzustellen, darf bezweifelt werden; bemerkenswert ist aber, dass Grießhaber eine eher pessimistische Haltung einnimmt, wie sie von linguistischer Seite ansonsten kaum vertreten wird.

\subsection{Schnittstellen: öffentlicher und wissenschaftlicher Diskurs}

Öffentlicher und wissenschaftlicher Diskurs laufen nicht völlig getrennt voneinander, es kommt immer wieder zu Berührungspunkten. Schnittstellen ergeben sich einerseits, wenn im wissenschaftlichen Diskurs auf die Öffentlichkeit verwiesen wird, bspw. um deren Kulturpessimismus ad absurdum zu führen oder dem problematischen Verhältnis von Sprachwissenschaft und Sprachkritik nachzugehen (s. o.). ${ }^{17}$ Fachtagungen, bei denen auch die interessierte Öffentlichkeit geladen ist, stellen ebenfalls eine solche Verknüpfung dar. Andererseits gibt es Schnittstellen, wenn Wissenschaftler in den öffentlichen Diskurs treten, bspw. indem sie selbst im öffentlichen Diskurs publizieren oder zitiert werden - besonders Letzteres ist häufig der Fall. Auf wissenschaftliche Studien wird bevorzugt verwiesen, um eine Aussage zu belegen und ihr mehr Stichhaltigkeit zu verleihen. Dies führt das folgende Zitat beispielhaft vor, das auf die Jahrestagung des Instituts für Deutsche Sprache (IDS) im März 2005 Bezug nimmt:

\footnotetext{
16 Vgl. Antos 1996 und Schrodt 2003.

17 Vgl. bspw. Trabold (1993), Neuland (1996), Eichhoff-Cyrus/Hoberg (2000) und Spitzmüller et al. (2002).
} 
Junge Menschen, die nur über SMS kommunizierten, blieben in ihrer Grammatik äußerst beschränkt, ihnen fehle zudem die Fähigkeit, komplexe Texte zu verstehen, beklagten Wissenschaftler bei einer Fachtagung Ende vergangener Woche in Mannheim. (Handelsblatt 29.03.2005) ${ }^{18}$

Grundsätzlich lässt sich beobachten, dass Referenzen dieser Art, in denen der Aspekt 'Schreibkompetenz und neue Medien' und daran anknüpfend die schriftliche Alltagskommunikation derart negativ beurteilt wird, die Ausnahme sind. In der Regel werden mit Verweis auf wissenschaftliche Studien eher optimistische oder zumindest den Medienpessimismus relativierende Argumente gestützt. Die kritische Aussage von Ludwig Eichinger, Direktor des IDS, "die Zunahme von per Handy verschickten Kurzmitteilungen beeinträchtig[e] die Sprachfähigkeit von jungen Leuten" (Stern 18.03.2005) wird denn auch im selben Artikel relativiert mit seiner Ansicht, "das so genannte 'Simsen' sei 'eher positiv' zu sehen", da es zum Schreiben animiere. Dasselbe gilt auch für die skeptischen Äußerungen des Soziolinguisten Norbert Dittmar zum ethnolektalen Deutsch. Dittmar wird in einem Zeitungsartikel mit den folgenden Worten zitiert:

Ich wird phonetisch zu 'isch', Artikel werden weggelassen, hier und da auch lokale Präpositionen wie 'in', 'nach' oder 'zu'", erklärt er. [...] Dittmar hält das für problematisch, "denn einige dieser Jugendlichen werden Teile dieser Sprache auch als Erwachsene weiterbenutzen". (Focus Schule 5, 01.09.2007)

Unmittelbar im Anschluss daran werden Dittmars Aussagen in Frage gestellt. Dies geschieht mit Verweis auf ein Forschungsprojekt zur Jugendsprache, das unter der Leitung von Eva Neuland durchgeführt wurde:

Das sieht Eva Neuland, Professorin und Soziolinguistin an der Universität Wuppertal, anders.

[...] Dass die Kiezsprache das Hochdeutsche verhunzt, hält Neuland für unwahrscheinlich.

'Immer wenn mit einer Sprache gespielt wird, zeugt das von Kreativität und Innovationsfreude'.

(Focus Schule 5, 01.09.2007).

Wie dieses Beispiel auch deutlich macht, ist es nicht so sehr die Schreibkompetenz der Jugendlichen, die im Mittelpunkt der öffentlichen Diskussion steht, als vielmehr der Sprachgebrauch. Meist wird der öffentlich-gesellschaftlichen Sorge um den Sprachverfall durch Computer und Fernsehen mit Verweisen auf wissenschaftliche Studien begegnet. Wissenschaftlern (nicht nur Sprachwissenschaftlern) wird dabei einerseits besondere Beurteilungskompetenz zugesprochen (was sich an den Interviews ablesen lässt), andererseits nehmen Wissenschaftler auch selbst diese Kompetenz für sich in Anspruch, indem sie initiativ Texte verfassen und bei Äußerungen im öffentlichen Diskurs auf ihren beruflichen Hintergrund verweisen.

Bemerkenswerterweise sind jedoch die Sprachwissenschaftler selbst im öffentlichen Diskurs über das Schreiben in den neuen Medien eher unterrepräsentiert. Dies mag daran liegen, dass "das Interesse an der Sprache [...] in vielen Wissenschaften, aber auch in anderen Bereichen sehr groß, das Bestreben, linguistische Erkenntnisse aufzunehmen und mit Sprachwissenschaftlern in einen Dialog zu treten, äußerst gering" ist, wie Hoberg (1997: 55) postuliert. Andererseits tut sich die Sprachwissenschaft schwer damit, ihren Forschungsgegenstand öffentlichkeitsgerecht zu vermarkten, auf das öffentliche Interesse an Sprachfragen einzugehen und bestimmte Sprachbedürfnisse zu befriedigen (vgl. Hoberg 1997: 61). Dies führt dazu, dass zwischen dem öffentlichen und sprachwissenschaftlichen Diskurs zum Thema 'Schreiben und neue Medien' nur wenige diskursive Bezüge vorhanden sind und weitest-

\footnotetext{
${ }^{18}$ Die Tagung hatte das Thema "Text - verstehen. Grammatik und darüber hinaus". Allerdings ist weder dem IDS-Jahrbuch noch dem Tagungsbericht zu entnehmen, aus welchem Kontext die Aussage stammt, auf die im Handelsblatt verwiesen wird.
} 
gehend andere Fachbereiche, wie die Medienwissenschaft oder die Didaktik, die Schnittstellen zwischen Öffentlichkeit und Wissenschaft herstellen.

Abschließend sei ein Beispiel angeführt, in dem versucht wird, Laien sprachwissenschaftliche Erkenntnisse näher zu bringen. Es ist dies das kürzlich erschienene Duden-Büchlein "Vernäht und zugeflixt! Von Versprechern, Flüchen, Dialekten \& Co.". Dieses Buch, verfasst von den Journalistinnen Ilse Achilles und Gerda Pighin, entstand in Zusammenarbeit der Dudenredaktion mit der Deutschen Gesellschaft für Sprachwissenschaft (DGfS). Wissenschaftlich begleitet wurden die einzelnen Kapitel von Mitgliedern der DGfS, deren Namen auf der vorletzten Umschlagseite aufgelistet sind. So zeichnet sich Nanna Fuhrhop für das Kapitel "Vom richtigen Schreiben" verantwortlich. In diesem Kapitel finden sich Erläuterungen zum Schreiben in den neuen Medien, und es werden charakteristische Beispiele (Abkürzungen, Akronyme) aufgelistet. Im Anschluss daran schreiben die Autorinnen:

Auch wenn es immer wieder heißt, die Jugend beherrsche ihre Muttersprache kaum noch, muss man sich um die Chatter und Simser keine ernsthaften Sorgen machen, vorausgesetzt, die Ergebnisse einer Studie der Universität von Coventry lassen sich auf deutsche Verhältnisse übertragen. (Achilles/Pighin 2008: 64)

In diesem Kapitel wird also explizit auf die Besorgnis um den Sprachgebrauch Jugendlicher eingegangen und in diesem Zusammenhang auf die Ergebnisse einer wissenschaftlichen Studie verwiesen. Weiter erfährt der Leser, dass es sich dabei um eine Studie mit 11-jährigen Schülern handelt, die ergab, dass "Heranwachsende, die viel chatten und simsen, einen viel besseren Wortschatz haben als ihre nicht chattenden Mitschüler" (Achilles/Pighin 2008: 64). Allerdings sei kritisch angemerkt, dass keine Quellenverweise und auch keine weiteren Informationen zu der Untersuchung gegeben werden. Wie unsere Recherchen aber ergaben, basiert die Studie auf einer Befragung von 35 Schülern. ${ }^{19}$ Es stellt sich also nicht nur die Frage, ob die Ergebnisse auf deutsche Verhältnisse übertragbar sind, sondern mehr noch, ob sie überhaupt als Evidenz herangezogen werden können. Zumindest wirft es ein kritisches Licht auf unsere Disziplin, wenn auf Fragen, die die Öffentlichkeit bewegen, in einem DudenBand auf diese Weise eingegangen wird.

\subsection{Schreiben in den neuen Medien - Segen oder Fluch?}

In den vorangehenden Abschnitten wurde dargelegt, dass Linguisten Stellung zu der Frage beziehen müssen, wie das Schreiben in den neuen Medien zu beurteilen ist, ob es also Segen oder Fluch (oder womöglich beides zugleich) ist. Dass sich hier Veränderungen abzeichnen, dass sprachkritische Fragen auch von Fachvertretern diskutiert werden, zeigt sich programmatisch - im Bozner Manifest (vgl. Fußnote 2); und es zeigt sich ebenfalls in neueren fachwissenschaftlichen Beiträgen. Dies bestätigt beispielsweise ein Blick in das Inhaltsverzeichnis der Zeitschrift Aptum, in der sprachkritische Fragen im Zentrum stehen. ${ }^{20}$ Und auch ein bekannter Medienlinguist wie Androutsopoulos (2007: 93) stellt fest: "Die künftige linguistische Internetforschung wird [...] nicht umhin kommen, überzeugende Antworten auf die öffentlich geäußerten Ängste vor eventuell 'schädlichen' Auswirkungen digitaler auf außerdigitale, institutionelle Schriftlichkeit anzubieten." Er weist in diesem Zusammenhang

\footnotetext{
19 Diese Information konnten wir nur aus Zeitungsartikeln über die Studie entnehmen, so z. B. aus einem Artikel in der Times (09.09.2006), auf den immer wieder verwiesen wird. Eine Publikation zu dieser Studie scheint nicht vorzuliegen, und auch auf der Website von Beverly Plester, die die Untersuchung durchgeführt hat und im Jahr 2006 bei der Jahrestagung der British Psychological Society darüber referierte, finden sich keine näheren Angaben.

${ }^{20}$ Nicht unerwähnt bleiben darf allerdings, dass einer der beiden Herausgeber der Zeitschrift Aptum, Jürgen Schiewe, selbst Unterzeichner des Bozner Manifests ist. Er setzt also die in diesem Text vorgetragenen Forderungen praktisch um.
} 
darauf hin, dass es beispielsweise sinnvoll sein könne, die Schreibpraxis von Schülerinnen und Schülern empirisch zu überprüfen.

Was Jannis Androutsopoulos einfordert, geschieht bereits, eine empirische Untersuchung ist im Gange, und zwar im Rahmen des vom Schweizerischen Nationalfonds geförderten Forschungsprojekts 'Schreibkompetenz und neue Medien' (vgl. zu einem Kurzbericht Wagner 2007), an dem die Autorinnen dieses Beitrags maßgeblich beteiligt sind. Nach unseren bisherigen Erkenntnissen kommt es nicht zu Interferenzen zwischen den verschiedenen Formen des Schreibens. Mit anderen Worten: Jugendliche, die in ihrer Freizeit häufig informell schreiben, haben im Deutschunterricht dadurch keinen Nachteil - aber auch keinen Vorteil, da an ihr schulisches Schreiben andere Erwartungen gestellt werden. So darf in einem Schulaufsatz konzeptionelle Mündlichkeit allenfalls als Stilmittel eingesetzt werden, und es wird orthographisch korrektes Schreiben verlangt. Wir nehmen also an - und erste Ergebnisse bestätigen dies -, dass die Schüler durchaus die verschiedenen Anwendungsdomänen zu unterscheiden wissen und ihre SMS anders schreiben als ihre Deutschaufsätze. Detaillierte Forschungsergebnisse liegen allerdings noch nicht vor; mit ihrer Publikation ist im Laufe des Jahres 2010 zu rechnen (vgl. Dürscheid/Wagner/Brommer i. V.).

Doch auch wenn zum jetzigen Zeitpunkt noch keine abschließenden Aussagen dazu möglich sind, ob die neue Alltagskommunikation tatsächlich - pointiert gesagt - keinen Fluch darstellt, was bereits feststeht, ist: Für die Linguistik ist sie ein Segen. Denn die neue, medienbasierte Schriftlichkeit bietet die Chance, vorhandene Theorien und Methodologien auf neue Daten zu beziehen, den bisherigen Theoriestand zu reflektieren und gegebenenfalls neue Ansätze zu entwickeln. Welche dies sein könnten, wird im nächsten Abschnitt gezeigt.

\section{$4 \quad$ Linguistische Analysen}

\subsection{Das Mündlichkeits-/Schriftlichkeitsmodell}

Wenden wir uns also abschließend der Frage zu, in welchem theoretischen Rahmen getippte Dialoge analysiert werden können. Nach unserer Überzeugung darf man sich dabei nicht auf die sprachliche Analyse der Beiträge im Instant Messaging beschränken. Auch der situative Kontext muss berücksichtigt werden, denn nur dann wird man den spezifischen Bedingungen, unter denen sich diese Form der schriftlichen Kommunikation vollzieht (und die in der Regel unter Bekannten stattfindet), gerecht. Die Gesprächsanalyse, die den Schwerpunkt auf die Beschreibung der Äußerungssequenzen selbst legt, kommt deshalb nicht in Frage. Ein prominenter Ansatz, auf den sich die Medienlinguistik der letzten zehn Jahre immer wieder beruft, ist das Mündlichkeits-/Schriftlichkeitsmodell von Koch/Oesterreicher (1994), auf das auch hier bereits Bezug genommen wurde. Dieses macht einen Unterschied zwischen medialer und konzeptioneller Mündlichkeit resp. Schriftlichkeit. Damit stellt das Modell eine präzise Terminologie bereit und bietet einen konzeptionellen Rahmen, in dem SMS-, Chattexte und E-Mails zueinander und zu herkömmlichen Texten in Beziehung gesetzt werden können. Es gibt jedoch mindestens vier Kritikpunkte, die dazu Anlass geben, sich nach einem alternativen Beschreibungsansatz umzusehen:

1) Jannis Androutsopoulos (2007: 79) stellt in seinem Beitrag 'Neue Medien - neue Schriftlichkeit?' in Bezug auf das Modell von Koch/Oesterreicher (1994) fest: "Es gehört zu den Paradoxien der deutschsprachigen linguistischen Internetforschung, sich eines Ansatzes zu bedienen, der noch vor dem Siegeszug der Neuen Medien konzipiert wurde und diese nicht einmal am Rande berücksichtigt." In der Tat ist hier ein Anachronismus zu beobachten, der auch dann bestehen bleibt, wenn, wie in Dürscheid (2003) geschehen, versucht wird, das Modell so zu modifizieren, dass auch Äußerungsformen aus der computer- und handyvermittelten Kommunikation erfasst werden können. 
2) Ein weiteres Problem liegt darin, dass der Gebrauch des Terminus 'medial' (vgl. 'mediale Schriftlichkeit') Verwirrung stiftet, da in der Medienlinguistik unter 'medial' etwas anderes verstanden wird als im Modell von Koch/Oesterreicher (1994). Diese beziehen den Ausdruck lediglich auf die beiden Repräsentationsformen von Sprache (geschrieben vs. gesprochen), legen also einen sehr engen Medienbegriff zugrunde, während in der Medienlinguistik 'medial' primär im Sinne von 'mittels eines technischen Hilfsmittels' verwendet wird.

3) Der dritte Kritikpunkt betrifft die Tatsache, dass das Modell kein Instrumentarium bereitstellt, mit dem kommunikative Aktivitäten sequenziell untersucht werden könnten. So ist es zwar geeignet, wenn es um die Einordnung von Äußerungsformen im Mündlichkeits-/ Schriftlichkeitskontinuum geht und die sprachlichen Merkmale dieser Äußerungsformen bestimmt werden sollen. Kommunikativ-situative Aspekte lassen sich auf diese Weise aber nicht analysieren. Das dagegen leistet die Interaktionale Linguistik, die im Folgenden vorgestellt werden soll.

4) Will man dem Prozess schriftlicher Kommunikation gerecht werden, dann genügt es nicht, die Dialogsequenzen im Rahmen des Mündlichkeits-/Schriftlichkeitsmodells zu analysieren, da dieses primär statisch ausgerichtet ist und sich an dem Produkt selbst orientiert. Das mag im Übrigen (neben der Sprachbarriere) auch ein Grund dafür sein, warum das Modell in der englischsprachigen Literatur kaum rezipiert wird. Viele Forschungsarbeiten situieren sich hier im Kontext der 'Computer-Mediated Discourse Analysis' (vgl. Herring 2001) - und damit in einem theoretischen Rahmen, der dem nun folgenden Plädoyer für die Interaktionale Linguistik sehr nahe kommt.

\subsection{Die Interaktionale Linguistik}

Ziel der interaktionalen [sic] Linguistik ist es, sequenziell interpretierte Redebeiträge, Turnkonstruktionseinheiten und Äußerungen in ihre sprachlichen Bestandteile zu zerlegen, um festzustellen, wie einzelne sprachliche Hinweise zu Praktiken wie der Bildung von Einheiten, Redebeiträgen und Handlungen beitragen, und zu zeigen, inwiefern diese Hinweise interaktional relevant sind. (Selting/Couper-Kuhlen 2000: 9)

Mit diesen Worten charakterisieren Margret Selting und Elizabeth Couper-Kuhlen eine linguistische Disziplin, an deren Konstituierung sie selbst maßgeblich beteiligt waren. Die Interaktionale Linguistik, so schreiben sie weiter, beginne immer mit der Untersuchung empirischer Daten, d. h. mit der Analyse situationsgebundener Sprache zwischen Gesprächsteilnehmern in natürlicher Interaktion, und sie betrachte die sprachlichen Einheiten in dieser "Rede-in-der-Interaktion" (Selting/Couper-Kuhlen 2000: 4) nicht isoliert, sondern als das Ergebnis interaktionaler Handlungen. Im Fokus der Interaktionalen Linguistik steht also das Gespräch. Doch lassen sich alle diese Aussagen auch auf die neuen Formen der schriftlichen Interaktion beziehen: Auch hier liegen empirische Daten vor (z. B. Chatmitschnitte), auch hier handelt es sich um eine natürliche Interaktion, in der die Äußerungen situationsgebunden sind und die Beteiligten den Sinngehalt der Äußerungen gemeinsam konstruieren, und auch hier beziehen sich die Beiträge wechselseitig aufeinander. Dieses 'Schreiben-in-derInteraktion' unterliegt zwar anderen Bedingungen als das Face-to-Face-Gespräch, aber anders als z. B. Briefe und Postkarten weist es auch wichtige Gemeinsamkeiten mit einem Gespräch auf. Schauen wir uns dazu einen kleinen Ausschnitt aus dem Instant-Messaging-Dialog der zwei Studenten an, deren 'Unterhaltung' weiter oben schon in Auszügen wiedergegeben wurde:

(1) A: heute prüfung gehabt

(2) B: oh

(3) A: gestern begonnen zu lernen

(4) B: und? 
(2) und (4) sind knappe, responsive Sequenzen, mit denen B unmittelbar auf die vorangehenden Äußerungen von A eingeht. Sie dienen dazu, Interesse an den Äußerungen von A zu bekunden, Empathie zu zeigen und eine Information zum Verlauf der Prüfung zu erhalten. Dabei ist die Frage "und?" im gegebenen Kontext vollkommen verständlich; ein vollständiger Satz ist hier nicht erforderlich. ${ }^{21}$ Ein solcher Dialog könnte ebenso in einem Face-to-FaceGespräch stattfinden - und würde dann im Untersuchungshorizont der Interaktionalen Linguistik liegen.

Will man nun - und dafür plädieren wir - auch die schriftliche Kommunikation, sofern sie quasi-synchron verläuft, in diesem Rahmen untersuchen, dann gibt es zwei Herangehensweisen: Entweder bilden "a) sprachliche Formen den Ausgangspunkt und es wird nach der Systematik ihrer Verwendung gefragt" (Deppermann 2007: 17), oder es wird "b) eine konversationelle Aufgabe [...] identifiziert und die linguistischen Formen ihrer Bearbeitung werden untersucht" (ebd.). ${ }^{22}$ Die Studie von Günthner (2000) zu den Vorwurfsaktivitäten im Alltag lässt sich dem zweiten Untersuchungsbereich zuordnen. Als Forschungsschwerpunkte, die zum ersten Untersuchungsbereich gehören, nennt Arnulf Deppermann, der selbst in diesem Rahmen arbeitet, 1) Prosodie und Rhythmus, 2) Diskursmarker und Konnektoren, 3) syntaktische Strukturen und 4) Code-Switching. Es ist evident, dass die genannten Phänomene - abgesehen von Prosodie und Rhythmus - ebenso auf schriftliche Dialoge bezogen werden können. So lassen sich auch hier Diskursmarker und Konnektoren analysieren und syntaktische Strukturen herausfiltern, die als typisch für die gesprochene Sprache gelten (vgl. "weil ich bin nach wie vor der meinung das ich jeden streber noch easy in den sack stecke" 23 ).

Allerdings muss auch eingestanden werden, dass aufgrund des Fehlens prosodischer Phänomene in der schriftlichen Kommunikation ein wichtiger Faktor wegfällt. In der Tat ist es ja so, dass in Gesprächen gerade die Prosodie an der Bedeutungskonstitution beteiligt ist. So schreibt Susanne Günthner (2000: 128) in der bereits erwähnten Arbeit, dass die "vorwurfsvolle Stimme" maßgeblich zur Kontextualisierung von Vorwürfen beiträgt. Doch es gibt in der schriftlichen Interaktion andere Gestaltungsprinzipien, die an der Bedeutungskonstitution mitwirken. Diese sind typographischer Art. Dazu gehören nicht nur die bekannten Strategien emulierter Prosodie (vgl. Wie schöööön!!), sondern auch typographische Verfahren, die, wie Androutsopoulos (2007: 83f.) treffend schreibt, "die Visualität von Schrift indexikalisch ausnutzen" (z. B. Schriftart, Schriftgröße, Schriftfarbe und andere Möglichkeiten typographischer Hervorhebung).

Ein Grund dafür, warum die schriftliche Kommunikation in der Interaktionalen Linguistik bislang nicht berücksichtigt wurde, ist zweifellos der folgende: Ihr Erkenntnisinteresse sei, so schreibt Günthner (2000: 2), herauszuarbeiten, welche Verfahren von "Interagierenden eingesetzt werden, um den Sinngehalt einer Äußerung erkennbar zu machen bzw. zu erkennen." Vergleichbares ist in der herkömmlichen Brief-, Postkarten- und Zettelkommunikation natürlich nicht möglich. Hier gibt es keine direkte Interaktion, in der der "Sinngehalt einer Äußerung" ausgehandelt werden könnte. Neu ermöglichen nun aber das OnlineSchreiben und das Schreiben am Handy auch in der Schriftlichkeit ein unmittelbares,

\footnotetext{
${ }^{21}$ Eine elliptische Frage, die unmittelbar an eine vorangehende Äußerung anknüpft, ist charakteristisch für Konstruktionen, die Wolfgang Klein (1993) unter dem Stichwort Adjazenzellipsen beschrieben hat (vgl. auch die folgende Sequenz aus dem oben bereits erwähnten IM-Dialog: "ich lerne viel zu wenig - ich auch").

22 Deppermann spricht fälschlich von "linguistischen" Formen. Gemeint sind sprachliche Formen, also Ausdrucksmittel, mit denen eine konversationelle Aufgabe bewältigt wird. Eine solche Verwechslung von 'linguistisch' (auf die Sprachbeschreibung bezogen) und 'sprachlich' (auf die Sprache bezogen) kommt häufig vor. Es ist vermutlich eine Interferenz aus dem Englischen, wo nur der Ausdruck linguistic gebräuchlich ist (vgl. linguistic theory vs. linguistic skills).

23 Das Beispiel stammt aus dem IM-Dialog, der weiter oben in Auszügen abgedruckt ist.
} 
interaktives Handeln. Das freilich ist nur dann der Fall, wenn die Technologie in einem entsprechenden "Interaktionsrhythmus" (Terminus von Androutsopoulos 2007: 89) genutzt wird, wenn es sich also, wie im Chat der Fall, um eine Kommunikation handelt, in der die Beiträge quasi-synchron aufeinander folgen. Zwar treten in diesen Dialogen nicht alle für die Bedeutungskonstitution relevanten Ausdrucksmittel auf, doch es gibt alternative Ausdrucksformen, die wiederum nur in schriftlichen Interaktionen vorkommen. Wenn auch diese in der Interaktionalen Linguistik erfasst würden, dann wäre das nicht nur eine erhebliche Erweiterung ihres Untersuchungsgegenstandes, sondern auch ihres Forschungshorizonts.

\section{$5 \quad$ Resümee}

Wer als Linguist zum Schreiben in den Medien forscht und dabei das dialogische Schreiben im Blick hat, der wird je nach Forschungsperspektive einen anderen Schwerpunkt wählen: Wer von der Textlinguistik her kommt, wird vielleicht die Unterschiede zum herkömmlichen Schreiben von Texten fokussieren, wer sich an der Gesprächslinguistik orientiert, wird getippte Dialoge mit Face-to-Face-Gesprächen vergleichen wollen, wer medienlinguistisch arbeitet, wird möglicherweise das Schreiben im Kontext anderer Ausdrucksformen in den neuen Medien untersuchen. Allen diesen Herangehensweisen gemeinsam ist, dass sie bestrebt sind, die Daten adäquat zu beschreiben. Das war auch unser Anliegen, als wir in Abschnitt 2 die verschiedenen Facetten des Handy-, Online- und Offline-Schreibens vorgestellt und die Unterschiede herausgearbeitet haben.

Was viele Linguisten in der Regel aber nicht tun, ist, den von ihnen beschriebenen Datenbereich negativ zu bewerten, also im konkreten Fall die Auffassung zu vertreten, dass dieses Schreiben ein 'schlechtes' Schreiben sei und dass negative Auswirkungen damit verbunden sein könnten. Auch wir haben das nicht getan, wir haben aber in Abschnitt 3 die damit verbundene Diskussion, wie sie in der Öffentlichkeit geführt wird, rekapituliert und darauf hingewiesen, dass empirische Untersuchungen nötig sind, um die Einflüsse des neuen Schreibens auf das herkömmliche Schreiben beurteilen zu können. Gleichzeitig war es uns ein Anliegen, darauf hinzuweisen, dass sprachkritische Einschätzungen zum Schreiben in den neuen Medien Ernst genommen werden müssen und Linguisten in dieser Frage das Terrain nicht nur Laien überlassen dürfen. In diesem Zusammenhang haben wir auch dafür plädiert, dass Linguisten sich mit ihren Publikationen stärker an die Öffentlichkeit wenden sollten. Dass hier noch einiges im Argen liegt, hat ein kritischer Blick auf das Duden-Bändchen "Vernäht und zugeflixt!" gezeigt.

Nach diesen Ausführungen zur Rolle der Linguistik im öffentlichen Diskurs wurde in Abschnitt 4 wieder die fachinterne Perspektive eingenommen. Im Zentrum standen die folgenden Fragen: Wie kann das Schreiben in den neuen Medien, das Führen von Dialogen über die Tastatur, linguistisch adäquat beschrieben werden? Welche Analysemethoden sind hierfür geeignet, welche nicht? Dabei machten wir deutlich: Will man der Dynamik getippter Dialoge gerecht werden, dann genügt es nicht, Äußerungsformen dieser Art im Rahmen des Modells von Koch/Oesterreicher (1994) zu betrachten. Ein Forschungsansatz, der sich als geeigneter erweist, ist die Interaktionale Linguistik. Weiteren Untersuchungen bleibt es vorbehalten, dies am Datenmaterial selbst, an Auszügen aus schriftlichen Interaktionen in den neuen Medien, zu erproben. Worum es uns primär ging, war, den Blick des Lesers auf die Meta-Ebene zu richten, also der Frage nachzugehen, welche linguistischen Beschreibungsansätze geeignet sein können, um getippte Dialoge theoretisch und methodologisch zu erfassen. Denn eines steht fest: Getippte Dialoge sind weder prototypische Gespräche noch prototypische Texte. Sie lassen sich also weder mit den Mitteln der Gesprächslinguistik noch mit denen der Textlinguistik vollständig beschreiben. 


\section{Literatur}

Achilles, Ilse/Pighin, Gerda (2008): Vernäht und zugeflixt! Von Versprechern, Flüchen, Dialekten \& Co. Mannheim etc.: Dudenverlag.

Antos, Gerd (1996): Laien-Linguistik. Studien zu Sprach- und Kommunikationsproblemen im Alltag. Am Beispiel von Sprachratgebern und Kommunikationstrainings. Tübingen. (= Reihe Germanistische Linguistik 146).

Androutsopoulos, Jannis (2007): "Neue Medien. Neue Schriftlichkeit?" Mitteilungen des Germanistenverbandes 54/1: Medialität und Sprache: 72-97.

Bittner, Johannes (2003): Digitalität, Sprache, Kommunikation. Eine Untersuchung zur Medialität von digitalen Kommunikationsformen und Textsorten und deren varietätenlinguistischer Modellierung. Berlin.

Brommer, Sarah (2006): Der öffentliche Diskurs über die Schreibkompetenz der Jugendlichen. Eine diskurskritische Analyse von Zeitungs- und Zeitschriftentexten. Magisterarbeit. Albert-Ludwigs-Universität Freiburg.

Brommer, Sarah (2007): "'Ein unglaubliches Schriftbild, von Rechtschreibung oder Interpunktion ganz zu schweigen'. Die Schreibkompetenz der Jugendlichen im öffentlichen Diskurs". Zeitschrift für germanistische Linguistik 35/3: 315-345.

Deppermann, Arnulf (2007): Grammatik und Semantik aus gesprächsanalytischer Sicht. Berlin. (= Linguistik - Impulse und Tendenzen 14).

Dürscheid, Christa (2003): "Medienkommunikation im Kontinuum von Mündlichkeit und Schriftlichkeit. Theoretische und empirische Probleme". Zeitschrift für Angewandte Linguistik 38: 37-56.

Dürscheid, Christa (2004): "Netzsprache. Ein neuer Mythos". Beißwenger, Michael/Hoffmann, Ludger/Storrer, Angelika (ed.) (2004): Internetbasierte Kommuni-kation. Osnabrück: 141-157. (= Osnabrücker Beiträge zur Sprachtheorie 68).

Dürscheid, Christa/Wagner, Franc/Brommer, Sarah (i.V.): Schreibkompetenz und neue Medien. Theoretische und empirische Aspekte.

Eichhoff-Cyrus, Karin M./Hoberg, Rudolf (eds.) (2000): Die deutsche Sprache zur Jahrtausendwende. Sprachkultur oder Sprachverfall? Mannheim.

Freyermuth, Gundolf (2002): Kommunikette 2.0. - E-Mail, Handy \& Co richtig einsetzen. Mit Top-Regeln für den digitalen Alltag. Hannover.

Gauger, Hans-Martin (1999): "Die Hilflosigkeit der Sprachwissenschaft". In: Meier, Christian (ed.): Sprache in Not? Zur Lage des heutigen Deutsch. Göttingen: 86-101.

Gauger, Hans-Martin (2004): Was wir sagen, wenn wir reden. Glossen zur Sprache. München/Wien.

Grießhaber, Wilhelm (2007): "Deutsch im Umbruch. Zu einigen Aspekten von Sprachwandel im Sprachkontakt". In: Redder, Angelika (ed.): Diskurse und Texte. Festschrift für Konrad Ehlich zum 65. Geburtstag. Tübingen: 339-344.

Günthner, Susanne (2000): Vorwurfsaktivitäten in der Alltagsinteraktion. Grammatische, prosodische, rhetorisch-stilistische und interaktive Verfahren bei der Konstitution kommunikativer Muster und Gattungen. Tübingen. (= Reihe Germanistische Linguistik 221).

Hayes, John R./Flower, Linda S. (1980): "Identifying the organization of writing processes". In: Gregg, Lee. W./Steinberg, Edwin R. (eds.): Cognitive Processes in Writing. Hillsdale: 3-30.

Herring, Susan C. (2001): "Computer-mediated Discourse". In: Schiffrin, Deborah/Tannen, Deborah/Hamilton, Heidi E. (eds.): The Handbook of Discourse Analysis. Oxford: 612634.

Hoberg, Rudolf (1997): "Öffentlichkeit und Sprachwissenschaft". Muttersprache. Vierteljahresschrift für Deutsche Sprache 107/1: 54-63. 
Jakobs, Eva-Maria/Jechle, Thomas (1995): "Veränderte Anforderungen an Schreiben und Schriftlichkeit". Diskussion Deutsch 26/141: 53-61.

Jakobs, Eva-Maria/Merker-Melcher, Ines (2003): "Sprachproduktion im Kontext medialer Individualkommunikation". In: Herrmann, Theo/Grabowski, Joachim (eds.): Sprachproduktion. Göttingen etc.: 825-845. (= Enzyklopädie der Psychologie C III Bd. 1).

Kessler, Florence (2008): "Instant Messaging. Eine neue interpersonale Kommunikationsform". Networx 52.

http://www.mediensprache.net/de/networx/docs/network-52.asp (Stand 26.01.2009).

Klein, Wolfgang (1993): "Ellipse". In: Jacobs, Joachim/Sternefeld, Wolfgang von/Vennemann, Theo (eds.): Syntax. Ein internationales Handbuch zeitgenössischer Forschung. Berlin: 763-799.

Koch, Peter/Oesterreicher, Wulf (1994): "Schriftlichkeit und Sprache". In: Günther, Hartmut/ Ludwig, Otto (eds.): Schrift und Schriftlichkeit. Ein interdisziplinäres Handbuch internationaler Forschung. Berlin: 587-604.

Lanthaler, Franz et al. (2003): "Sprachkritik und Sprachwissenschaft - Anmerkungen zu einer komplizierten Beziehung". Sprachreport 19/2: 2-5.

Neuland, Eva (1996): "Sprachkritiker sind wir doch alle! Formen öffentlichen Sprachbewusstseins. Perspektiven kritischer Deutung und einige Folgerungen". In: Böke, Karin/Jung, Matthias/Wengeler, Martin (eds.): Öffentlicher Sprachgebrauch. Praktische, theoretische und historische Perspektiven. Festschrift Georg Stötzel. Opladen: 110-121.

Raible, Wolfgang (2001): "Wohin steuert unsere Sprache? Diagnosen und Prognosen an der Jahrtausendwende". In: Panagl, Oswald/Goebl, Hans/Brix, Emil (eds.): Der Mensch und seine Sprache. Wien/Köln/Weimar 1-23.

Schlobinski, Peter (2003): "SMS-Texte - Alarmsignale für die Standardsprache?" Sprachrohr Lerntherapie 2/2003: 27-28.

http://www.mediensprache.net/de/essays/2/ (Stand 26.01.2009).

Schlobinski, Peter (ed.) (2006): Von *hdl* bis *cul8er*. Sprache und Kommunikation in den neuen Medien. Mannheim.

Schneider, Jan Georg (2005): "Was ist ein sprachlicher Fehler? Anmerkungen zu populärer Sprachkritik am Beispiel der Kolumnensammlung Bastian Sick". Aptum. Zeitschrift für Sprachkritik und Sprachkultur 2/2005: 154-177.

Schrodt, Richard (2003): "Das Sprachbarometer zeigt Stur: Nutzen und Notwendigkeit soziologischer Konzepte bei der Erforschung der deutschen Gegenwartssprache". In: Betten, Anne/Schrodt, Richard/Weiss, Andreas (eds.): Neue Sprachmoral? Medien, Politik, Schule. Wien 15-23. (= Stimulus. Mitteilungen der Österreichischen Gesellschaft für Germanistik).

Selting, Margret/Couper-Kuhlen, Elizabeth (2000): "Argumente für die Entwicklung einer interaktionalen Linguistik". Gesprächsforschung - Online-Zeitschrift zur verbalen Interaktion. 1/2000: 76-95.

http://www.gespraechsforschung-ozs.de/heft2000/ga-selting.pdf (Stand 26.01.2009).

Spinnen, Burkhard (1992): "'... unser Schreibzeug arbeitet mit an unseren Gedanken'. Anmerkungen zum Computerschreiben". Sprache im technischen Zeitalter 121/1992: 4152.

Spitzmüller, Jürgen et al. (eds.) (2002): Streitfall Sprache. Sprachkritik als angewandte Linguistik? Bremen. (= Freiburger Beiträge zur Linguistik 3).

Spitzmüller, Jürgen (2006): "Der mediale Diskurs zu 'Jugendsprache': Kontinuität und Wandel". In: Dürscheid, Christa/Spitzmüller, Jürgen (eds.): Perspektiven der Jugendsprachforschung. Trends and Developments in Youth Language Research. Frankfurt am Main: 33-50. (= Sprache - Kommunikation - Kultur. Soziolinguistische Beiträge 3).

Storrer, Angelika (2001): "Getippte Gespräche oder dialogische Texte? Zur kommunikationstheoretischen Einordnung der Chat-Kommunikation". In: Lehr, Andrea/Kammerer, 
Matthias et al. (eds.): Sprache im Alltag. Beiträge zu neuen Perspektiven in der Linguistik. Herbert Ernst Wiegand zum 65. Geburtstag gewidmet. Berlin etc.: 439-465.

Trabold, Annette (1993): Sprachpolitik, Sprachkritik und Öffentlichkeit. Anforderungen an die Sprachfähigkeit des Bürgers. Wiesbaden.

Wagner, Franc (2007): "Das Zürcher Projekt 'Schreibkompetenz und neue Medien'". Zeitschrift für germanistische Linguistik 35/3: 466-470.

Ziefle, Martina (1998): "Effects of Display Resolution on Visual Performance". Human Factors 40/4: 554-568.

Zimmer, Dieter E. (2005): Sprache in Zeiten ihrer Unverbesserlichkeit. Hamburg.

\section{Verzeichnis der zitierten Zeitungs- und Zeitschriftentexte}

Die Texte sind chronologisch aufgelistet mit Angabe zu Datum, Zeitung, Verfasser und vollständigem Titel. Die Autorenkürzel wurden soweit möglich aufgelöst.

Frankfurter Allgemeine Zeitung (13.08.1997) Klaus, Martin: "Alter Fisch in neuen Netzen".

Süddeutsche Zeitung (27.10.1997) Rheinz, Hanna: "Die Kollision von Schrift und Sprache oder: Wie der PC den Schreibstil verändert".

Zeit (20.08.1998) Benning, Maria: "Schreib, wie du sprichst".

Frankfurter Allgemeine Zeitung (31.07.2001) Schmoll, Heike: "Sprachverwirrung".

Frankfurter Allgemeine Zeitung (28.02.2002) Kaube, Jürgen: "Realitäts Prinzip".

Zeit (12.06.2003) Gaschke, Susanne: "Wer lesen will, muss hören".

Stern (20.01.2004) Barthélémy, Andrea, dpa: "Taste statt Füller: Eine neue Schriftkultur setzt sich durch".

Zeit (21.10.2004) Isler, Thomas/Puntas Bernet, Daniel: "Die Globalisierung des Simsens".

Stern (18.03.2005) dpa/AP: "Besser SMS schreiben als gar nichts".

Times Online (09.09.2006) Alexandra Frean: "Y txtng cn b v gd 4 improving linguistic ability of children".

http://www.timesonline.co.uk/tol/news/uk/artilcle633544.ece (Stand 26.01.2009).

Spiegel (05.05.2006) Patalong, Frank: "Falsch ist das neue Richtig".

Focus Schule (01.0.9.2007) Sven Trojanowski: "Red isch Deutsch oda was?". http://www.focus.de/schule/lernen/bildung-red-isch-deutsch-oda-was_aid_222259.html (Stand 26.01.2009).

SonntagsZeitung (18.11.2007) Simone Luchetta: "Lachen heisst heute LOL". 